\title{
D branes in background fluxes and Nielsen-Olesen instabilities
}

\author{
Jorge G. Russo \\ Institució Catalana de Recerca i Estudis Avançats (ICREA), \\ Pg. Lluis Companys, 23, 08010 Barcelona, Spain \\ Department de Fisica Cuantica i Astrofisica and Institut de Ciències del Cosmos, \\ Universitat de Barcelona, Martí Franquès, 1, 08028 Barcelona, Spain \\ E-mail: jorge.russo@icrea.cat
}

ABSTRACT: In quantum field theory, charged particles with spin $\geq 1$ may become tachyonic in the present of magnetic fluxes above some critical field, signaling an instability of the vacuum. The phenomenon is generic, in particular, similar instabilities are known to exist in open and closed string theory, where a spinning string state can become tachyonic above a critical field. In compactifications involving RR fluxes $F_{p+2}$, the quantum states which could become tachyonic by the same Nielsen-Olesen mechanism are $\mathrm{D} p$ branes. By constructing an appropriate background with $\mathrm{RR}$ magnetic flux that takes into account back-reaction, we identify the possible tachyonic $\mathrm{D} p$ brane states and compute the formula for the energy spectrum in a sector. More generally, we argue that in any background $\mathrm{RR}$ magnetic flux, there are high spin $\mathrm{D} p$ quantum states which become very light at critical fields.

Keywords: D-branes, Flux compactifications, Superstrings and Heterotic Strings

ARXIV EPRINT: 1604.03484 


\section{Contents}

1 Introduction 1

2 Fundamental string in NS-NS 3-form flux $H_{3}$

3 Dp branes in the $\boldsymbol{F}_{p+2}$ background $\quad 7$

4 Discussion 11

\section{Introduction}

In some theories, turning on magnetic fields can lead to Nielsen-Olesen instabilities [1, 2]. They occur for charged particles with spin. Their origin can be simply understood from the energy formula for a charged particle in a uniform magnetic field $B$ :

$$
E^{2}=M^{2}+q B(2 \ell+1)-2 q B S,
$$

where $M$ is the mass, $q$ is the charge, $S$ is the spin, we took the gyromagnetic factor $g=2$, and $\ell=0,1,2, \ldots$ is the Landau level. For a scalar particle, $S=0$ and the gyromagnetic interaction contributes positively to the energy. For a spin $1 / 2$ fermion, the minimum energy occurs when $S=1 / 2$, in which case $E^{2}=M^{2}$. However, for a spin 1 particle, such as the $W_{ \pm}$bosons, the component with spin parallel to the magnetic field, $S=1$, would have

$$
E^{2}=M^{2}-q B
$$

A tachyon instability appears if $q B>M^{2}$. This leads to condensation of $W$ bosons (which are then expected to form a triangular lattice of vortices, $[3,4]$ ). More generally, a spin $S$ particle with spin aligned to the magnetic field, becomes tachyonic if

$$
E^{2}=M^{2}-q B(g|S|-1)<0 \quad \text { or } \quad q B>\frac{M^{2}}{g|S|-1} .
$$

In string theory, there are particles with all spins. This implies that the negative contribution coming from the gyromagnetic interaction can be large, even for very weak magnetic fields. For a given mass, the maximum spin scales like $M^{2}$, so the gyromagnetic interaction competes with the rest mass term. As a result, the value of the critical magnetic field depends on the detailed form of the energy formula. However, the general lesson is that we should expect Nielsen-Olesen instabilities in any theory with spinning objects which are charged under some flux that is turned on. This applies to strings, D strings and, more generally, $\mathrm{D} p$ branes, which can also have arbitrarily large spin.

Nielsen-Olesen instabilities have been studied for open strings [5-7] and for closed strings [8-12] in various contexts. In this paper we study, for the first time, this phenomenon 
in $\mathrm{D}$ branes. Different aspects of $\mathrm{D}$ branes in background $\mathrm{RR}$ magnetic fluxes have been discussed in [13]. Here we will use the same magnetic background. In the presence of a $F_{p+2}=d A_{p+1}$ background magnetic flux, the energy of $\mathrm{D} p$ branes gets reduced for some angular momentum components. Understanding the emergence of instabilities requires identifying potential tachyonic modes and a precise determination of the energy formula.

This paper is organized as follows. In section 2 we study tachyon instabilities in the superstring spectrum in the presence of an $\mathrm{H}_{3}$ flux, in the context of an exact conformal string $\sigma$ model. The mass spectrum exhibits the appearance of tachyons above critical fields. We show that some of these tachyon states admit a semiclassical description in terms of classical string solutions moving in the background flux. We close this section by discussing a supersymmetry-preserving field configuration and the corresponding formula for the mass spectrum. In section 3 we consider the dynamics of $\mathrm{D} p$ branes moving in a background with $F_{p+2}$ flux. We identify a classical $\mathrm{D} p$ brane solution which becomes tachyonic above a critical field. Finally, in section 4, we give a more general discussion on instabilities induced by generic flux configurations, in particular, for quantized fluxes on cycles. We briefly comment on the implications for the construction of metastable flux compactifications with broken supersymmetry.

\section{Fundamental string in NS-NS 3-form flux $\boldsymbol{H}_{3}$}

It is useful to start with strings in $H_{3}$ fluxes, as in this case we can determine the complete energy spectrum. We consider the following string $\sigma$ model

$$
\begin{aligned}
L= & -\partial_{+} t \partial_{-} t+\partial_{+} \rho \partial_{-} \rho+F(\rho) \rho^{2}\left(\partial_{+} \varphi+b \partial_{+} y\right)\left(\partial_{-} \varphi-b \partial_{-} y\right) \\
& +\partial_{+} y \partial_{-} y+\partial_{+} x^{i} \partial_{-} x^{i}+\frac{1}{4} \alpha^{\prime} \sqrt{g} R^{(2)}\left(\phi_{0}+\frac{1}{2} \ln F\right), \quad F=\frac{1}{1+b^{2} \rho^{2}} .
\end{aligned}
$$

with $i=1, \ldots, 6$. It represents an exact conformal field theory for type II superstrings. We use the conventions $\sigma^{ \pm}=\tau \pm \sigma$ and $\sigma \in[0,2 \pi)$. $y$ is a periodic coordinate, $y=y+2 \pi R$. For simplicity, here we only quote the bosonic part. It is straightforward to incorporate the world-sheet fermions [9]. The Lagrangian (2.1) describes strings propagating in the geometry

$$
\begin{aligned}
d s^{2} & =-d t^{2}+d \rho^{2}+\rho^{2} F(\rho) d \varphi^{2}+F(\rho) d y^{2}+d x_{i}^{2}, \\
e^{2\left(\phi-\phi_{0}\right)} & =F, \quad B_{2}=b \rho^{2} F(\rho) d \varphi \wedge d y .
\end{aligned}
$$

This string model is obtained, by T-duality in the $y$ direction, from the Kaluza-Klein "Melvin" string sigma model [9]

$$
\begin{aligned}
L= & -\partial_{+} t \partial_{-} t+\partial_{+} \rho \partial_{-} \rho+\rho^{2}\left(\partial_{+} \varphi+\tilde{b} \partial_{+} y\right)\left(\partial_{-} \varphi+\tilde{b} \partial_{-} y\right) \\
& +\partial_{+} y \partial_{-} y+\partial_{+} x^{i} \partial_{-} x^{i}
\end{aligned}
$$

with constant dilaton and vanishing $B_{2}$. The $\sigma$-model (2.3) describes a locally flat spacetime, as can be seen by a formal redefinition of the polar angle, $\varphi^{\prime}=\varphi+\tilde{b} y$. Because $y$ is 
periodic, the solution of the model is non-trivial. The model (2.3) can be solved in terms of free fields by carefully imposing the appropriate boundary conditions.

It is convenient to introduce complex coordinates $x=\rho e^{i \varphi}$. Clearly, $\rho e^{i \varphi^{\prime}}$ is a free field and the general solution is given by [9]

$$
\begin{aligned}
& x=e^{-i \tilde{b} y(\tau, \sigma)}\left(X_{+}\left(\sigma^{+}\right)+X_{-}\left(\sigma^{-}\right)\right), \\
& y=y_{+}\left(\sigma_{+}\right)+y_{-}\left(\sigma_{-}\right) .
\end{aligned}
$$

One can choose world-sheet coordinates such that

$$
y_{+}\left(\sigma_{+}\right)+y_{-}\left(\sigma_{-}\right)=y_{0}+w R \sigma+\alpha^{\prime} p_{y} \tau
$$

where $w$ is the winding number and $p_{y}=n / R, n \in \mathbb{Z}$ is the quantized Kaluza-Klein momentum. Demanding that $x$ is single-valued, $x(\sigma+2 \pi, \tau)=x(\sigma, \tau)$, leads to twisted boundary conditions for free fields $X^{+}, X^{-}$,

$$
X(\tau, \sigma+2 \pi)=e^{2 \pi i \gamma} X(\tau, \sigma), \quad \gamma \equiv \tilde{b} w R
$$

Hence

$$
X_{ \pm}=e^{ \pm i \gamma \sigma_{ \pm}} \chi_{ \pm}\left(\sigma_{ \pm}\right),
$$

where $\chi_{ \pm}\left(\sigma_{ \pm}\right)$are single-valued free fields, with the Fourier expansion

$$
\chi_{+}=i \sqrt{\frac{\alpha^{\prime}}{2}} \sum_{n=-\infty}^{\infty} \tilde{a}_{n} e^{-i n \sigma^{+}}, \quad \chi_{-}=i \sqrt{\frac{\alpha^{\prime}}{2}} \sum_{n=-\infty}^{\infty} a_{n} e^{-i n \sigma^{-}} .
$$

The $\sigma$-model (2.1), while equivalent as a conformal field theory to the T-dual model (2.3), describes strings moving in curved space-time and as a result the general solution to the string equations of motion is more complicated. It can be obtained from section 5 in [8], adapting the parameters to the present case. The general solution is

$$
\begin{aligned}
x & =e^{-i b\left(y_{+}\left(\sigma_{+}\right)-y_{-}\left(\sigma_{-}\right)\right)}\left(X_{+}\left(\sigma^{+}\right)+X_{-}\left(\sigma^{-}\right)\right), \\
y & =y_{+}\left(\sigma_{+}\right)+y_{-}\left(\sigma_{-}\right)-b \tilde{\varphi}, \\
\partial_{ \pm} \tilde{\varphi} & = \pm \frac{i}{2}\left(X^{*} \partial_{ \pm} X-X \partial_{ \pm} X^{*}\right) .
\end{aligned}
$$

Note that

$$
y_{+}\left(\sigma_{+}\right)-y_{-}\left(\sigma_{-}\right)=w R \tau+\alpha^{\prime} p_{y} \sigma
$$

$X_{+}, X_{-}$satisfy the same twisted boundary conditions as before, now with

$$
\gamma=b \alpha^{\prime} p_{y} .
$$

Note, however, that $y$, given by (2.10), is not a free field in this case. 
Mass spectrum and tachyon instabilities. The mass spectrum for the model (2.1) is given by the formula

$$
\begin{aligned}
\alpha^{\prime} M^{2} & =2 \hat{N}_{R}+2 \hat{N}_{L}+\frac{\alpha^{\prime} n^{2}}{R^{2}}+\frac{R^{2}}{\alpha^{\prime}}\left(w-b \frac{\alpha^{\prime}}{R} \hat{J}\right)^{2}-2 \gamma\left(\hat{J}_{R}-\hat{J}_{L}\right), \\
\hat{N}_{R}-\hat{N}_{L} & =n w, \quad \gamma=\frac{\alpha^{\prime} n b}{R} .
\end{aligned}
$$

Here $\hat{N}_{R}, \hat{N}_{L}$ have the standard free string theory expression in terms of normal-ordered bi-linear products of bosonic and fermionic oscillators,

$$
\hat{N}_{R, L}=N_{R, L}-a, \quad a^{(\mathrm{R})}=0, \quad a^{(\mathrm{NS})}=\frac{1}{2} .
$$

Thus $\hat{N}_{R, L}$ have eigenvalues $0,1, \ldots$ (half-integer eigenvalues are projected out by GSO). The angular momentum operators are

$$
\hat{J}_{L, R}= \pm\left(l_{L, R}+\frac{1}{2}\right)+S_{L, R}, \quad \hat{J}=\hat{J}_{L}+\hat{J}_{R}=l_{L}-l_{R}+S_{L}+S_{R}
$$

and $l_{L, R}=0,1,2, \ldots$ are Landau quantum numbers and the spin components $S_{L, R}$ have the same expressions as in free string theory and satisfy the bound $\left|S_{L, R}\right| \leq \hat{N}_{L, R}+1$. The mass formula is periodic with $\gamma$. As written, the formula applies for $|\gamma|<1$; in other intervals one must substitute $\gamma \rightarrow \hat{\gamma}=\gamma-[\gamma]$.

We note that there are two gyromagnetic couplings in (2.13): one involves the product of the winding charge $w$ and $\hat{J}_{R}+\hat{J}_{L}$; the other one involves the product of the KaluzaKlein charge $n$ and $\hat{J}_{R}-\hat{J}_{L}$. Equivalently, the Left charge $Q_{L}=\frac{w R}{\alpha^{\prime}}+\frac{n}{R}$ couples to $J_{R}$, the Right charge $Q_{R}=\frac{w R}{\alpha^{\prime}}-\frac{n}{R}$ couples to $J_{L}$.

The mass spectrum for the string model (2.1) contains tachyons for a flux above some critical value, which are not projected out by GSO. The first tachyon appears for the state

$$
\begin{aligned}
& \hat{N}_{R}=\hat{N}_{L}=0, \quad S_{R}=1, \quad S_{L}=-1, \quad w=0, \quad n=1, \\
& l_{R}=l_{L}=0, \quad \hat{J}=0, \quad \hat{J}_{R}-\hat{J}_{L}=1, \quad \gamma=b \frac{\alpha^{\prime}}{R} .
\end{aligned}
$$

Then

$$
M^{2}=\frac{1}{R^{2}}-2 \frac{b}{R}
$$

which is tachyonic for $b>b_{\mathrm{cr}}, b_{\mathrm{cr}}=1 /(2 R)$. Now, taking into account the condition $\gamma<1$, the tachyon can appear only when $R^{2}>\alpha^{\prime} / 2$.

Let us now consider states with high quantum numbers, $\hat{N}_{R}$ or $\hat{N}_{L} \gg 1$. In particular, we consider the state

$$
\begin{aligned}
& \hat{N}_{L}=\hat{N}_{R}, \quad w=0, \quad p_{y}=n / R, \\
& l_{R}=l_{L}=0, \quad S_{R}=-S_{L}=\hat{N}_{R}+1, \\
& \hat{J}=0, \quad \hat{J}_{R}-\hat{J}_{L}=2 \hat{N}_{R}+1 .
\end{aligned}
$$


These are states with maximum left and right components of the spin, chosen in a way that the negative contribution to the energy coming from the gyromagnetic interaction involving $\hat{J}_{R}-\hat{J}_{L}$ is maximized. Substituting these quantum numbers into the mass formula, we get

$$
\begin{aligned}
\alpha^{\prime} M^{2} & =4 \hat{N}_{R}+\frac{\alpha^{\prime} n^{2}}{R^{2}}-2 \gamma\left(2 \hat{N}_{R}+1\right) \\
& =4 \hat{N}_{R}(1-\gamma)+\frac{\alpha^{\prime} n^{2}}{R^{2}}-2 \gamma \\
& =\frac{\alpha^{\prime} n^{2}}{R^{2}}+2(1-\gamma)\left(\hat{J}_{R}-\hat{J}_{L}\right)-2, \quad \gamma=\frac{\alpha^{\prime} n b}{R} .
\end{aligned}
$$

This is tachyonic for

$$
\gamma>\frac{4 \hat{N}_{R}+\alpha^{\prime} n^{2} / R^{2}}{4 \hat{N}_{R}+2} .
$$

The condition $\gamma<1$ is satisfied with $n^{2}<2 R^{2} / \alpha^{\prime}$. All these states become tachyonic above the critical field (2.18).

Classical string solutions associated with instabilities. It is instructive to identify the classical string solution corresponding to the tachyonic states with $\hat{N}_{R}=\hat{N}_{L} \gg 1$, for which a semiclassical description should apply. Since the state has maximum value for the spin components, this state is obtained by setting to zero all oscillator modes $a_{n}, \tilde{a}_{n}$ with $|n| \neq 1$, and turning on $\tilde{a}_{ \pm 1}, a_{ \pm 1}$ such that the solution has maximum $\hat{J}_{R}-\hat{J}_{L}$, with vanishing total $\hat{J}=\hat{J}_{R}+\hat{J}_{L}$, i.e. the same quantum numbers as in (2.16). Note that non-zero Landau levels, coming from $a_{0}, \tilde{a}_{0}$, give an (orbital) contribution to the angular momentum in the antiparallel direction and they increase the energy.

The left and right angular momentum components are given by the following formulas (see $(5.29)$ in [8])

$$
J_{R}=-\frac{1}{2} \sum_{n}(n+\gamma) a_{n}^{*} a_{n}, \quad J_{L}=-\frac{1}{2} \sum_{n}(n-\gamma) \tilde{a}_{n}^{*} \tilde{a}_{n} .
$$

The classical string with the same angular momentum components as the quantum state (2.16) thus has $\tilde{a}_{1} \neq 0$ and $a_{-1} \neq 0$, setting all other modes to zero. This gives

$$
J_{R}=\frac{1}{2}(1-\gamma) a_{-1}^{*} a_{-1}, \quad J_{L}=-\frac{1}{2}(1-\gamma) \tilde{a}_{1}^{*} \tilde{a}_{1},
$$

with $\left|a_{-1}\right|^{2}=\left|\tilde{a}_{1}\right|^{2}$. Therefore, the solution (2.9), (2.12), with $w=0$, takes the form

$$
x=e^{-i b \alpha^{\prime} p_{y} \sigma}\left(X_{+}+X_{-}\right)
$$

where

$$
\begin{aligned}
& X_{+}=i \sqrt{\frac{\alpha^{\prime}}{2}} e^{i \gamma \sigma_{+}} \tilde{a}_{1} e^{-i \sigma_{+}}=L e^{-i(1-\gamma) \sigma_{+}}, \\
& X_{-}=i \sqrt{\frac{\alpha^{\prime}}{2}} e^{-i \gamma \sigma_{-}} a_{-1} e^{i \sigma_{-}}=L e^{i(1-\gamma) \sigma_{-}} .
\end{aligned}
$$

That is,

$$
x=2 L e^{-i \sigma} \cos [(1-\gamma) \tau]
$$


This represents a circular pulsating string. The classical description applies for $L \gg l_{s}$, where $l_{s} \equiv \sqrt{\alpha^{\prime}}$.

Using (2.10), (2.11), we also find

$$
y=\alpha^{\prime} p_{y} \tau-b L^{2}(2(1-\gamma) \tau+\sin [2(1-\gamma) \tau]) .
$$

In order to compute the energy, we have to solve the Virasoro constraints $T_{++}=T_{--}=0$. This leads to the formula

$$
\begin{aligned}
\alpha^{\prime} p_{0}^{2} & =\alpha^{\prime} p_{y}^{2}+\frac{4 L^{2}}{\alpha^{\prime}}(1-\gamma)^{2} \\
& =\frac{\alpha^{\prime} n^{2}}{R^{2}}+2(1-\gamma)\left(J_{R}-J_{L}\right)
\end{aligned}
$$

This agrees with the quantum formula (2.17) except for the -2 contribution. This is a small quantum contribution, which is not visible in the semiclassical approximation, which requires $\hat{N}_{R} \gg 1$ and thus $4 \hat{N}_{R}+2 \approx 4 \hat{N}_{R}$. In the classical limit, the critical field gives $\gamma \rightarrow 1$ (see (2.18)). As a result, the energy — which for zero field is large - attains a minimum value $p_{0}=n / R$. The tachyon instability is not seen classically, but we know that it exists, because we have computed the exact quantum spectrum. Classically, the sign that there is an instability at a critical field is the fact that, as the magnetic field is increased, the energy decreases from a very large, macroscopic value $l_{s} p_{0}=2 \sqrt{N_{R}}=2 L / l_{s} \gg 1$ to a tiny value $O\left(l_{s} / R\right)$, i.e. to a value that may be overcome by a negative quantum contribution of $O\left(1 / l_{s}\right)$.

Supersymmetric extension and mass spectrum. The background (2.2) breaks all supersymmetries. A way to preserve a fraction of the supersymmetries is by introducing several magnetic fields in different planes [14]. For example, consider the solution obtained by introducing two fluxes, one in the plane $\left(r_{1}, \varphi_{1}\right)$ and the other in the plane $\left(r_{2}, \varphi_{2}\right)$. The background is as follows

$$
\begin{aligned}
d s^{2} & =-d t^{2}+d x_{i}^{2}+d r_{1}^{2}+d r_{2}^{2}+r_{1}^{2} d \varphi_{1}^{2}+r_{2}^{2} d \varphi_{2}^{2}+\Lambda^{-1}\left(d y-\left(b_{1} r_{1}^{2} d \varphi_{1}+b_{2} r_{2}^{2} d \varphi_{2}\right)^{2}\right) \\
e^{-2\left(\phi-\phi_{0}\right)} & =\Lambda, \quad B_{2}=\Lambda^{-1}\left(b_{1} r_{1}^{2} d \varphi_{1}+b_{2} r_{2}^{2} d \varphi_{2}\right) \wedge d y \\
\Lambda & =1+b_{1} r_{1}^{2}+b_{2} r_{2}^{2} .
\end{aligned}
$$

The corresponding string sigma model is exactly conformal and solvable, and the mass spectrum is given by [14]

$$
\begin{aligned}
\alpha^{\prime} M^{2}= & 2\left(\hat{N}_{R}+\hat{N}_{L}\right)+\frac{R^{2}}{\alpha^{\prime}}\left(w-b_{1} \frac{\alpha^{\prime}}{R} \hat{J}_{1}-b_{2} \frac{\alpha^{\prime}}{R} \hat{J}_{2}\right)^{2}+\alpha^{\prime} \frac{n^{2}}{R^{2}} \\
& -2 \alpha^{\prime} b_{1} \frac{n}{R}\left(\hat{J}_{1 R}-\hat{J}_{1 L}\right)-2 \alpha^{\prime} b_{2} \frac{n}{R}\left(\hat{J}_{2 R}-\hat{J}_{2 L}\right),
\end{aligned}
$$

with $\hat{N}_{R}-\hat{N}_{L}=n w$. For $b_{1} \neq \pm b_{2}$, there are tachyonic instabilities appearing at critical fields. However, when $b_{1}= \pm b_{2}$, there are sixteen unbroken supersymmetries. In this case, 
one has $M^{2} \geq 0$ for all states in the spectrum, as expected from supersymmetry. Taking $b_{1}=b_{2} \equiv b$, the mass spectrum becomes

$$
M^{2}=\frac{2}{\alpha^{\prime}}\left(\hat{N}_{R}+\hat{N}_{L}\right)+\left(\frac{w R}{\alpha^{\prime}}-b\left(\hat{J}_{1}+\hat{J}_{2}\right)\right)^{2}+\frac{n^{2}}{R^{2}}-2 b \frac{n}{R}\left(\hat{J}_{1 R}+\hat{J}_{2 R}-\hat{J}_{1 L}-\hat{J}_{2 L}\right) .
$$

An important class of states are the BPS states, investigated in [13]. The BPS states have $\hat{N}_{L}=0\left(\right.$ or $\left.\hat{N}_{R}=0\right)$. Then the mass becomes a perfect square provided $\hat{J}_{1}+\hat{J}_{2}=$ $\hat{J}_{1 R}+\hat{J}_{2 R}-\hat{J}_{1 L}-\hat{J}_{2 L}$, which implies

$$
\left(S_{1 L}, S_{2 L}\right)=(-1,0) \text { or }(0,-1)
$$

Then

$$
M_{\mathrm{BPS}}^{2}=\left(\frac{n}{R}+\frac{w R}{\alpha^{\prime}}-b\left(\hat{J}_{1}+\hat{J}_{2}\right)\right)^{2} .
$$

Supersymmetry ensures that the energy formula is exact and does not receive quantum corrections. In the zero field limit, this gives the familiar formula, with $M_{\mathrm{BPS}}$ being the sum of winding and KK charges. Since $\alpha^{\prime} n b / R<1$, and $\hat{J}_{1}+\hat{J}_{2} \leq \hat{N}_{R}=n w$, the minimum mass is not zero, but $M_{\mathrm{BPS}}=n / R$. For $R \gg \sqrt{\alpha^{\prime}}$, this is a tiny, microscopic energy, compared with the energy at zero field, $M_{\mathrm{BPS}}=w R / \alpha^{\prime}+n / R$. This is a surprising feature, since the formula $\hat{N}_{R}=n w$ implies that, for $n w \gg 1$, this is highly excited string state. Moreover, the BPS state admits a semiclassical description in terms of a long, rotating string, where the same energy formula is reproduced [13]. This macroscopic string has a microscopic energy at a critical field.

\section{Dp branes in the $F_{p+2}$ background}

Consider the general problem of a $\mathrm{D} p$ brane moving on a background with $F_{p+2}$ flux. Assuming that there is no other field turned on, the $\mathrm{D} p$ brane action is given by

$$
S_{\mathrm{D} p}=-\tau_{p} \int d^{p+1} \sigma e^{-\left(\phi-\phi_{0}\right)} \sqrt{-\operatorname{det}\left(G_{a b}\right)}+i \tau_{p} \int A_{p+1}
$$

with

$$
\tau_{p}=\frac{1}{(2 \pi)^{p} l_{s}^{p+1} g_{s}}, \quad l_{s} \equiv \sqrt{\alpha^{\prime}} .
$$

Starting with (2.27), by S-duality we find a solution with $F_{3}=d A_{2}$ flux. By T-duality transformations along the different $x_{i}$, one can construct a solution with $F_{p+2}$ flux,

$$
\begin{aligned}
d s^{2}= & \Lambda^{\frac{1}{2}}\left(-d t^{2}+d x_{s}^{2}+d r_{1}^{2}+d r_{2}^{2}+r_{1}^{2} d \varphi_{1}^{2}+r_{2}^{2} d \varphi_{2}^{2}\right) \\
& +\Lambda^{-\frac{1}{2}}\left(d y_{1}^{2}+\ldots+d y_{p}^{2}-\left(b_{1} r_{1}^{2} d \varphi_{1}+b_{2} r_{2}^{2} d \varphi_{2}\right)^{2}\right) \\
A_{p+1}= & e^{-\phi_{0}} \Lambda^{-1}\left(b_{1} r_{1}^{2} d \varphi_{1}+b_{2} r_{2}^{2} d \varphi_{2}\right) \wedge d y_{1} \wedge \ldots \wedge d y_{p} \\
e^{2\left(\phi-\phi_{0}\right)}= & \Lambda^{\frac{3}{2}-\frac{p}{2}}, \quad \Lambda=1+b_{1} r_{1}^{2}+b_{2} r_{2}^{2},
\end{aligned}
$$

with $s=p+5, \ldots, 9$. 
Consider first the action (3.1) for the D string in the background (3.2) with $p=1$. One immediately finds that this coincides with the Nambu-Goto action for a fundamental string moving in the background (2.2). As the Lagrangians are the same, this has the important consequence that the solutions are the same and that the classical energies for any $\mathrm{D}$ string motion will be the same as in the fundamental string case, with the appropriate modification in the string tension (this arises because, after $\mathrm{S}$ duality, one must rescale time variable in string frame by a factor $g_{s}$ to have Minkowski metric in the zero field limit). ${ }^{1}$

In the supersymmetric $b_{1}= \pm b_{2}$ case, there is a $\mathrm{D} p$ brane analog of the BPS string state (2.30), studied in [13]. The rotating $\mathrm{D} p$ brane has essentially the same energy formula (2.30), replacing $w R / \alpha^{\prime}$ by $\tau_{p} \operatorname{Vol}\left(T_{p}\right) \omega$, where $\omega$ is the winding number of the $\mathrm{D} p$ brane around the torus $T_{p}$,

$$
M_{\mathrm{BPS}}=\frac{n}{R}+\left|\tau_{p} \operatorname{Vol}\left(T_{p}\right) \omega-b J\right| .
$$

In particular, the energy of this macroscopic $\mathrm{D} p$ brane decreases down to a tiny value $n / R$ at a critical field. At the critical field, the gyromagnetic coupling cancels the huge $\mathrm{D}$ brane energy $\tau_{p} \operatorname{Vol}\left(T_{p}\right) \omega$ due to tension.

For $b_{1} \neq \pm b_{2}$, the spectrum may contain tachyons above some critical fields, which will now be of order $1 / g_{s}$. Consider, for example, the non-supersymmetric solution with $b_{2}=0$, $b_{1} \equiv b$. The classical solution representing a pulsating $\mathrm{D} p$ brane, which is the analogue of the pulsating circular string solution (2.24), (2.25) considered above, can be constructed by starting with the following ansatz.

$$
t=\kappa \tau, \quad r_{1}=r(\tau), \quad \varphi=-\sigma_{1}, \quad y_{1}=y_{1}(\tau), \quad y_{j}=w_{j} R_{j} \sigma_{j}, \quad j=2, \ldots, p .
$$

The $\mathrm{D} p$ brane Lagrangian thus takes the form

$$
\mathcal{L}_{\mathrm{D} p}=-\frac{c_{p} r}{1+b^{2} r^{2}} \sqrt{\left(\kappa^{2}-\dot{r}^{2}\right)\left(1+b^{2} r^{2}\right)-{\dot{y_{1}}}^{2}}+\frac{c_{p} b r^{2} \dot{y}_{1}}{1+b^{2} r^{2}}
$$

with

$$
c_{p} \equiv(2 \pi)^{p} \tau_{p} R_{2} \ldots R_{p} w=\frac{R^{p-1} w}{l_{s}^{p+1} g_{s}}, \quad w=w_{2} \ldots w_{p} .
$$

The canonical momenta $p_{y}$ and $p_{r}$ are given by

$$
\begin{aligned}
& p_{y}=\frac{\partial \mathcal{L}_{\mathrm{D} p}}{\partial \dot{y}}=c_{p}\left(\frac{r \dot{y}}{\left(1+b^{2} r^{2}\right) \sqrt{\left(\kappa^{2}-\dot{r}^{2}\right)\left(1+b^{2} r^{2}\right)-\dot{y}^{2}}}+\frac{b r^{2}}{1+b^{2} r^{2}}\right) \\
& p_{r}=\frac{\partial \mathcal{L}_{\mathrm{D} p}}{\partial \dot{r}}=c_{p} \frac{r \dot{r}}{\sqrt{\left(\kappa^{2}-\dot{r}^{2}\right)\left(1+b^{2} r^{2}\right)-\dot{y}^{2}}}
\end{aligned}
$$

The Hamiltonian is then given by

$$
H=p_{r} \dot{r}+p_{y} \dot{y}-\mathcal{L}_{\mathrm{D} p}=c_{p} \frac{r \kappa^{2}}{\sqrt{\left(\kappa^{2}-\dot{r}^{2}\right)\left(1+b^{2} r^{2}\right)-\dot{y}^{2}}} .
$$

\footnotetext{
${ }^{1}$ The S-dual background (3.2) is not expected to be an exact solution to all $\alpha^{\prime}$ order. However, possible $\alpha^{\prime}$ corrections do not affect the energies of classical strings, which have lengths much greater than the string scale.
} 
One can express $\dot{y}$ and $\dot{r}$ in terms of $p_{y}$ and $p_{r}$,

$$
\begin{aligned}
& \dot{r}=\frac{\kappa p_{r}}{\sqrt{p_{y}^{2}+c_{p}^{2} r^{2}(1-\gamma)^{2}}}, \\
& \dot{y}=\frac{\kappa\left(p_{y}-c_{p} b r^{2}(1-\gamma)\right)}{\sqrt{p_{y}^{2}+c_{p}^{2} r^{2}(1-\gamma)^{2}}},
\end{aligned}
$$

where

$$
\gamma=\frac{b p_{y}}{c_{p}}
$$

Substituting these expressions into the Hamiltonian (3.7), after some algebra one finally obtains the surprisingly simple formula

$$
H=\kappa \sqrt{p_{r}^{2}+V(r)},
$$

with

$$
V(r)=p_{y}^{2}+c_{p}^{2} r^{2}(1-\gamma)^{2} .
$$

This Hamiltonian is dimensionless and generates translations in world-volume time $\tau$. The spacetime Hamiltonian is

$$
\mathcal{H}=\kappa^{-1} H=\sqrt{p_{r}^{2}+V(r)} ;
$$

it has as usual dimension of energy and generates translations in $t$.

The classical solution can be obtained by using energy conservation. It is similar to the circular string. We find

$$
r(\tau)=2 L \cos [(1-\gamma) \tau], \quad y=q \tau-b L^{2}(2(1-\gamma) \tau+\sin [2(1-\gamma) \tau]),
$$

with $\gamma=b q$, and $q=p_{y} / c_{p}$. The classical energy is then

$$
E^{2}=\frac{n^{2}}{R^{2}}+\frac{4 w^{2}}{g_{s}^{2}} \frac{R^{2 p-2} L^{2}}{l_{s}^{2 p+2}}(1-\gamma)^{2} .
$$

Again the energy attains a minimum value at $\gamma \rightarrow 1$ where the $\mathrm{D} p$ brane energy becomes tiny, equal to $p_{y}$ (rather than proportional to the size $L$ ). The difference with the BPS $\mathrm{D} p$ brane is that, in the BPS case, $E^{2}$ is garanteed to be greater or equal to zero by supersymmetry. For non-supersymmetric configurations, there is nothing that prevents $E^{2}$ from receiving a small, quantum negative correction, which can cause tachyon instabilities above a critical magnetic field.

Quantum energy. So far we have computed the classical energy (3.14). Although a complete quantization of the $p$-brane is not known for $p>1$, in the sector of the ansatz (where only a specific oscillation mode of the $\mathrm{D} p$ brane is turned on) we have a simple Hamiltonian and we can compute the quantized eigenvalues. 
Consider first the fundamental string, for which the exact quantum spectrum is known ${ }^{2}$ and whereby we can check the consistency of the present approach. In the Nambu-Goto formulation, the Hamiltonian is given by (3.11) with $c_{p} \rightarrow 2 \pi \tau_{1}=1 / \alpha^{\prime}$.

The eigenvalues of $H^{2}$ are well-known, since $H^{2} / 2$ is the Hamiltonian of the radial part of a 2-dimensional harmonic oscillator with frequency $\omega=|1-\gamma| / \alpha^{\prime}$. Assuming as before $\gamma<1$, we can remove absolute value bars. Therefore

$$
\alpha^{\prime} \mathcal{H}^{2}=\alpha^{\prime} E^{2}=\alpha^{\prime} p_{y}^{2}+2(2 k+1)(1-\gamma)+\delta, \quad k=0,1,2, \ldots
$$

where $k$ is radial quantum number. We have added a constant $\delta$ to account for possible normal ordering contributions, which cannot be computed from first principles in this sector. The reason is that the normal ordering constant picks contributions from all oscillators of the string, including all those set to zero in this sector. It is easy to compute $\delta$ in the zero field limit. In this limit, supersymmetry must be restored. A supersymmetric Hamiltonian must have zero energy in the ground state. This requires $\left.\delta\right|_{b=0}=-2$, so that $E=0$ for $k=0, p_{y}=0$.

Of course, the zero field limit does not determine what is $\delta$ at finite field $b . \delta$ could in general be $b$ dependent. One can determine $\delta$ by computing the ground state $(k=0)$ energy at finite field. In this case, the string is rigid, it has no excitation, and it is described by the supergravity multiplet. The energy of the graviton can be computed by using the effective field theory, i.e. solving the Laplace-type equation for the gravitational fluctuations in the magnetic background (2.2) (for a discussion, see [11]). For a state with $S_{R}=1, S_{L}=-1$, $p_{y}=1 / R$, this calculation leads to the same formula (2.15) found before for $\hat{N}_{R}=\hat{N}_{L}=0$, that we reproduce here

$$
E^{2}=\frac{1}{R^{2}}-2 \frac{b}{R}
$$

On the other hand, setting $k=0$ and $p_{y}=1 / R$ in our formula (3.15), we obtain

$$
\alpha^{\prime} E^{2}=\frac{1}{R^{2}}-2 \frac{b}{R}+2+\delta
$$

therefore $\delta=-2$ for all $b$. This matches (2.17),

$$
\alpha^{\prime} E^{2}=\alpha^{\prime} p_{y}^{2}+2(2 k+1)(1-\gamma)-2 .
$$

Note that the radial quantum number $k$ is related to $\hat{J}_{R}-\hat{J}_{L}$. Therefore we find the correct formula for the quantum spectrum in this sector.

We now generalize this calculation for a $\mathrm{D} p$ brane. The Hamiltonian (3.11), (3.12) is formally the same upon replacing $\alpha^{\prime}$ by $1 / c_{p}$. Thus, we obtain the eigenvalues:

$$
\begin{aligned}
E^{2} & =p_{y}^{2}+c_{p}\left(2(2 k+1)(1-\gamma)+\delta_{p}\right) \\
& =p_{y}^{2}-2 b p_{y}(2 k+1)+c_{p}\left(4 k+2+\delta_{p}\right), \quad \gamma=\frac{b p_{y}}{c_{p}}
\end{aligned}
$$

\footnotetext{
${ }^{2}$ Here, of course, we mean the exact quantum spectrum in $\alpha^{\prime}$; the quantum spectrum that includes string loops is not known even for flat spacetime. The regime of validity of the present approximations is $g_{s} \ll 1$ (or $g_{s} \gg 1$, in a dual description). That is, quantum field theory loops are not included.
} 
To compute $\delta_{p}$, we follow the same procedure as in the string case. Supersymmetry in the zero field limit requires, as before, $\left.\delta_{p}\right|_{b=0}=-2$. For finite $b$, we compute $\delta_{p}$ at for the ground state, $k=0$. This corresponds to a rigid $\mathrm{D} p$ brane, which can be dimensionally reduced to a D-string along the directions $y_{j}, j=2, \ldots, p$. The $k=0$ state then describes the state $S_{R}=1, S_{L}=-1$ having energy (3.16) (for this state, the energy does not depend on the tension; it corresponds to a point-like limit described by the effective field theory). This leads to $\delta_{p}=-2$ once again. Thus we have the general formula for quantum $\mathrm{D} p$ brane states in this sector:

$$
E^{2}=p_{y}^{2}+c_{p}(2(2 k+1)(1-\gamma)-2)
$$

i.e.

$$
E^{2}=p_{y}^{2}+\frac{R^{p-1} w}{l_{s}^{p+1} g_{s}}(2(2 k+1)(1-\gamma)-2) .
$$

At large $k$, the energy (3.21) agrees with the energy formula (3.14) of the classical pulsating D $p$ brane.

We see that a $\mathrm{D} p$ brane with quantum numbers $n, k, w$ becomes tachyonic above a critical field

$$
R b_{\mathrm{cr}}=\frac{n}{4 k+2}+\frac{2 k}{2 k+1} \frac{R^{p+1} w}{l_{s}^{p+1} g_{s} n} .
$$

Thus, as expected, a macroscopic $\mathrm{D} p$ brane can become tachyonic when magnetic fluxes are turned on.

\section{Discussion}

In this paper we have studied instabilities of the Nielsen-Olesen type in backgrounds with $\mathrm{RR}$ magnetic fluxes. In our examples, gravitational back reaction to the magnetic flux is incorporated exactly.

In compactifications having fluxes on cycles, fluxes are quantized in units of the inverse volume of the cycle and an interesting question is when to expect instabilities. The mere existence of a single tachyon in the quantum spectrum of a theory implies that the vacuum is unstable. It is obvious that, to linear order in the magnetic flux, the energy formula for a $\mathrm{D} p$ brane will contain the standard gyromagnetic interaction. Let us see this explicitly in a simple example. Let $x^{1}, \ldots, x^{p+2}$ be the coordinates of a rectangular torus, $T^{p+2}$. We consider the constant field configuration

$$
A_{p+1}=f x_{1} d x_{2} \wedge \ldots \wedge d x_{p+2} .
$$

Moreover, we choose a static gauge for the world-volume coordinates where $x_{j}=w_{j} R_{j} \sigma^{j}$, $j=3, \ldots, p+2$, and let us first assume that $x_{1}, x_{2}$ only depend on $\tau$. Then the coupling to the gauge field in the $\mathrm{D} p$ brane action becomes

$$
i \tau_{p}(2 \pi)^{p} R_{3} \ldots R_{p+2} w f \int d \tau x_{1} \partial_{\tau} x_{2} \equiv i w B \int d \tau x_{1} \partial_{\tau} x_{2}
$$

where $w=w_{3} \ldots w_{p+2}$. The flux quantization condition can be obtained by demanding that the generalized Wilson loop

$$
e^{i \tau_{p} \int A_{p+1}}
$$


is single-valued under $x_{1} \rightarrow x_{1}+2 \pi R_{1}$ (with periodic $\tau$ for a closed contour). This implies

$$
f=\frac{2 \pi k}{\tau_{p} \operatorname{Vol}\left(T^{p+2}\right)},
$$

where $k$ is an integer. This reproduces the result of [15]. Thus

$$
B=\frac{2 \pi k}{\operatorname{Vol}\left(T^{2}\right)}, \quad \operatorname{Vol}\left(T^{2}\right)=4 \pi^{2} R_{1} R_{2} .
$$

The interaction (4.2) exhibits a gyromagnetic coupling to the angular momentum of the brane in the plane $\left(x_{1}, x_{2}\right)$. In particular, when $p=1$, this is the same coupling that appears in open string theory (the open string mass spectrum for magnetic field in toroidal directions was computed in [6]). There is no rotational symmetry in the 12 plane, but as usual spin is defined for the Lorentz group acting on tangent space. Because we assumed that $x_{1}, x_{2}$ only depend on $\tau$, the brane has only orbital angular momentum. We can relax this assumption and let $x_{1}, x_{2}$ depend on some $\sigma^{i}$, in which case the brane will also carry spin and this term will give rise to the standard gyromagnetic interaction $w B(2 \ell+1-2 S)$. To linear order in the field $f$, for a brane in a quantum state of spin $S$, we would expect the mass formula to contain the terms

$$
\begin{aligned}
M^{2} & =M_{0}^{2}+\sum_{i} \frac{n_{i}^{2}}{R_{i}^{2}}+\left(w \tau_{p} \operatorname{Vol}\left(T^{p}\right)\right)^{2}-2 w \tau_{p} \operatorname{Vol}\left(T^{p}\right) f J_{12}+\ldots \\
& =M_{0}^{2}+\sum_{i} \frac{n_{i}^{2}}{R_{i}^{2}}+\left(w \tau_{p} \operatorname{Vol}\left(T^{p}\right)\right)^{2}+(2 \ell+1) B w-2 S B w+\ldots
\end{aligned}
$$

where $\ell=0,1,2, \ldots$ are the Landau levels and $M_{0}^{2}$ represents positive contributions originating from oscillations and kinetic energy of the brane. It must be noted that, for a $\mathrm{D} p$ brane, there are other linear couplings to the magnetic flux; even for the string, we have a coupling to $\hat{J}_{R}-\hat{J}_{L}$. Such coupling does not show up in this simple example because of the particular ansatz we took for the brane embedding (the coupling to $\hat{J}_{R}-\hat{J}_{L}$, related to $x_{1} \partial_{\sigma_{1}} x_{2}-x_{2} \partial_{\sigma_{1}} x_{1}$, appeared in the pulsating $\mathrm{D}$ brane discussed in section 3 ). The most general linear coupling to the flux is essentially read from (4.1) once the brane embedding is given.

However, this example gives an easy insight into some important features: 1) It exhibits the explicit dependence on the quantized flux in the gyromagnetic interaction. 2) In the exact quantum string spectrum (2.13), the gyromagnetic coupling involving the winding charge appears upon expanding a positive definite term. This exposes the fact that $O\left(B^{2}\right)$ terms are important in order to make a statement about the positivity of $M^{2}$. Determining such $O\left(B^{2}\right)$ terms requires taking into account the gravitational back reaction. This is a difficult problem in more general contexts, but the important point is that the linearized approximation already identifies the precise D brane states that can become light at critical fields. 3) Naively one might think that for sufficiently high spin, the term $2 S B w$ would render $M^{2}$ negative. But, of course, the angular momentum gives, in addition, a positive contribution to the kinetic energy, represented by $M_{0}^{2}$. In the string model, the 
oscillation/kinetic energy is represented by $2 \hat{N}_{L}+2 \hat{N}_{R}$, and the highest spin for a given kinetic energy is determined by the bound $\left|S_{L, R}\right| \leq \hat{N}_{L, R}+1$.

In our background magnetic flux, we have identified a tachyonic $\mathrm{D}$ brane state. It has zero angular momentum, but the linear coupling to the flux comes through $\hat{J}_{R}-\hat{J}_{L}$ (Right and Left being associated with $\left.\left(\partial_{\tau} \pm \partial_{\sigma_{1}}\right) \varphi\right)$. At large quantum numbers, it admits a semiclassical description in terms of a pulsating $\mathrm{D}$ brane. In general terms, even for supersymmetric configurations, where the spectrum is tachyon free and the vacuum is stable, we have seen that certain high angular momentum states become very light at critical fields. A naive field theory analysis would ignore such D brane quantum states, because they typically have huge energies of order $\tau_{p} \operatorname{Vol}\left(T_{p}\right) \omega$. However, we now see that at a certain critical field, such states can be very light, with energies $E \ll 1 / l_{s}$. Near the critical point, an effective field theory analysis should take into account these modes in order to properly describe the low energy dynamics of the system. ${ }^{3}$ More generally, for any given flux configuration, one can easily compute the classical energy formula to linear order in the fluxes to see if the spectrum contains a D brane state with high quantum numbers that became light by the interaction with the flux. In configurations with no residual supersymmetry, the appearance of $\mathrm{D} p$ brane tachyonic modes above some critical fields seems to be a common feature, by the same mechanism that induces Nielsen-Olesen instabilities in quantum field theory.

Non-supersymmetric flux compactifications have been extensively used for the construction of inflationary models and semi-realistic string compactifications [17]. In these models, fluxes generate a superpotential which freezes all Calabi-Yau moduli and supersymmetry is typically broken by the presence of anti-branes. An important question is whether some of these models could be affected by tachyonic instabilities of the kind studied in this paper. To address this problem, a direct approach is to use the $\mathrm{D} p$ brane Lagrangian (3.1) in the given background flux and to compute the classical energy formula - analogue to (4.6) - for a $\mathrm{D} p$ brane motion that maximizes the gyromagnetic interaction with low cost in oscillation/kinetic energy. Typically, these are rigidly rotating $\mathrm{D} p$ branes or pulsating Dp branes with no other oscillation modes turned on. A quick estimate of the expected order of the critical field can be obtained from (4.6), giving $f \sim \frac{w}{2 J} \tau_{p} \operatorname{Vol}\left(T^{p}\right){ }^{4}$ This is consistent with our examples, the BPS state with energy (3.3) or the pulsating $D p$ brane with critical field (3.22). At stronger fields, potential tachyonic instabilities can arise and the vacuum becomes unstable. This implies a bound for the maximum possible value for the quantized flux (4.4), roughly $k \lesssim \frac{c \tau_{p}^{2}}{4 \pi} \operatorname{Vol}\left(T^{p+2}\right) \operatorname{Vol}\left(T^{p}\right)$, where $c$ is a rational number (for our examples, $w / J$ or $w / n$ ), which in a first approximation may be set to $1 .^{5}$ It would be extremely interesting to revisit the counting of metastable string vacua with semi-realistic phenomenology (see e.g. [18]), in view of these new constraints on the possible choices of flux.

\footnotetext{
${ }^{3} \mathrm{~A}$ recent discussion on brane effective field theory in background fluxes can be found in [16].

${ }^{4}$ As discussed below (4.6), $J$ does not need to be the angular momentum.

${ }^{5}$ In particular, for an $H_{3}$ flux, this would give $k \lesssim\left(R / l_{s}\right)^{4}$.
} 


\section{Acknowledgments}

We would like to thank Arkady Tseytlin and Diego Rodriguez-Gomez for useful comments. We acknowledge financial support from projects FPA2013-46570, 2014-SGR-104 and MDM2014-0369 of ICCUB (Unidad de Excelencia 'María de Maeztu').

Open Access. This article is distributed under the terms of the Creative Commons Attribution License (CC-BY 4.0), which permits any use, distribution and reproduction in any medium, provided the original author(s) and source are credited.

\section{References}

[1] N.K. Nielsen and P. Olesen, An Unstable Yang-Mills Field Mode, Nucl. Phys. B 144 (1978) 376 [INSPIRE].

[2] J. Ambjørn and P. Olesen, On electroweak magnetism, Nucl. Phys. B 315 (1989) 606 [INSPIRE].

[3] J. Ambjørn and P. Olesen, A Magnetic Condensate Solution of the Classical Electroweak Theory, Phys. Lett. B 218 (1989) 67 [Erratum ibid. B 220 (1989) 659] [INSPIRE].

[4] J. Ambjørn and P. Olesen, A Condensate Solution of the Electroweak Theory Which Interpolates Between the Broken and the Symmetric Phase, Nucl. Phys. B 330 (1990) 193 [INSPIRE].

[5] S. Ferrara and M. Porrati, String phase transitions in a strong magnetic field, Mod. Phys. Lett. A 8 (1993) 2497 [hep-th/9306048] [InSPIRE].

[6] C. Bachas, A way to break supersymmetry, hep-th/9503030 [INSPIRE].

[7] J.G. Russo, Strong magnetic limit of string theory, JHEP 06 (2005) 005 [hep-th/0504187] [INSPIRE].

[8] J.G. Russo and A.A. Tseytlin, Exactly solvable string models of curved space-time backgrounds, Nucl. Phys. B 449 (1995) 91 [hep-th/9502038] [INSPIRE].

[9] J.G. Russo and A.A. Tseytlin, Magnetic flux tube models in superstring theory, Nucl. Phys. B 461 (1996) 131 [hep-th/9508068] [INSPIRE].

[10] E. Kiritsis and C. Kounnas, Infrared behavior of closed superstrings in strong magnetic and gravitational fields, Nucl. Phys. B 456 (1995) 699 [hep-th/9508078] [INSPIRE].

[11] J.G. Russo and A.A. Tseytlin, Magnetic backgrounds and tachyonic instabilities in closed superstring theory and M-theory, Nucl. Phys. B 611 (2001) 93 [hep-th/0104238] [INSPIRE].

[12] S. Minwalla and T. Takayanagi, Evolution of D-branes under closed string tachyon condensation, JHEP 09 (2003) 011 [hep-th/0307248] [INSPIRE].

[13] R. Iengo, J. Lopez Carballo and J.G. Russo, Strings and D-branes in a supersymmetric magnetic flux background, JHEP 08 (2007) 047 [arXiv:0707.0455] [INSPIRE].

[14] J.G. Russo and A.A. Tseytlin, Supersymmetric fluxbrane intersections and closed string tachyons, JHEP 11 (2001) 065 [hep-th/0110107] [INSPIRE].

[15] R. Bousso and J. Polchinski, Quantization of four form fluxes and dynamical neutralization of the cosmological constant, JHEP 06 (2000) 006 [hep-th/0004134] [INSPIRE]. 
[16] J. Polchinski, Brane/antibrane dynamics and KKLT stability, arXiv:1509.05710 [INSPIRE].

[17] S. Kachru, R. Kallosh, A.D. Linde and S.P. Trivedi, de Sitter vacua in string theory, Phys. Rev. D 68 (2003) 046005 [hep-th/0301240] [INSPIRE].

[18] M.R. Douglas, The statistics of string/M theory vacua, JHEP 05 (2003) 046 [hep-th/0303194] [INSPIRE]. 